\title{
Scale-up of room-temperature constructive quantum interference from single molecules to self-assembled molecular-electronic films
}

\author{
Xintai Wanga,e†, Troy L. R. Bennett ${ }^{\mathrm{b}, \dagger}$, Ali Ismael ${ }^{\mathrm{a}, \mathrm{c}, \dagger}$, Luke A. Wilkinson ${ }^{\mathrm{b}}$, Joseph Hamilld, An- \\ drew J. P. White ${ }^{b}$, Iain M. Grace ${ }^{a}$, Oleg V. Kolosov ${ }^{\mathrm{a}}$, Tim Albrecht ${ }^{\mathrm{d}}$, Benjamin J. Robinson ${ }^{\mathrm{a}^{*}}$, \\ Nicholas J. Long ${ }^{\mathrm{b}^{*}}$, Lesley F. Cohen ${ }^{\mathrm{e}^{*}}$ and Colin J. Lambert ${ }^{\mathrm{a}^{*}}$ \\ aPhysics Department, Lancaster University, Lancaster, LA1 4YB, UK. \\ bDepartment of Chemistry, Imperial College London, MSRH, White City, London, W12 oBZ, UK. \\ 'Department of Physics, College of Education for Pure Science, Tikrit University, Tikrit, Iraq. \\ ${ }^{\mathrm{d} D e p a r t m e n t ~ o f ~ C h e m i s t r y, ~ B i r m i n g h a m ~ U n i v e r s i t y, ~ E d g b a s t o n, ~ B i r m i n g h a m, ~ B 15 ~ 2 T T, ~ U K . ~}$ \\ eThe Blackett Laboratory, Imperial College London, South Kensington Campus, London, SW7 2AZ, UK.
}

\begin{abstract}
The realization of self-assembled molecularelectronic films, whose room-temperature transport properties are controlled by quantum interference (QI), is an essential step in the scale-up QI effects from single molecules to parallel arrays of molecules. Recently, the effect of destructive QI (DQI) on the electrical conductance of selfassembled monolayers (SAMs) has been investigated. Here, through a combined experimental and theoretical investigation, we demonstrate chemical control of different forms of constructive QI (CQI) in cross-plane transport through SAMs and assess its influence on cross-plane thermoelectricity in SAMs. It is known that the electrical conductance of single molecules can be controlled in a deterministic manner, by chemically varying their connectivity to external electrodes. Here, by employing synthetic methodologies to vary the connectivity of terminal anchor groups around aromatic anthracene cores, and by forming SAMs of the resulting molecules, we clearly demonstrate that this signature of CQI can be translated into SAM-ongold molecular films. We show that the conductance of vertical molecular junctions formed from anthracenebased molecules with two different connectivities differ by a factor of approximately 16 , in agreement with theoretical predictions for their conductance ratio based on constructive QI effects within the core. We also demonstrate that for molecules with thioether anchor groups, the Seebeck coefficient of such films is connectivity dependent and with an appropriate choice of connectivity can be boosted by $\sim 50 \%$. This demonstration of QI and its influence on thermoelectricity in SAMs represents a critical step towards functional ultra-thin-film devices for future thermoelectric and molecular-scale electronics applications.
\end{abstract}

Molecular electronic devices have the potential to deliver logic gates, sensors, memories and thermoelectric energy harvesters with ultra-low power requirements and sub-10 $\mathrm{nm}$ device footprints. ${ }^{1-4}$ Single-molecule electronic junctions ${ }^{5-12}$ and self-assembled monolayers ${ }^{13-15}$ have been investigated intensively over the past few years, because their room-temperature electrical conductance has been shown to be controlled by destructive quantum interference (DQI). ${ }^{16-20}$ More recently the effect of quantum interference on the Seebeck coefficient of single molecules has also been studied ${ }^{21-26}$. Figure $1(A)$ illustrates an example where a room-temperature constructive quantum interference (CQI) effect would be expected from an anthracene molecular core. Here, electrical current is injected into and collected from the core via the green arrows, or alternatively via the red arrows.

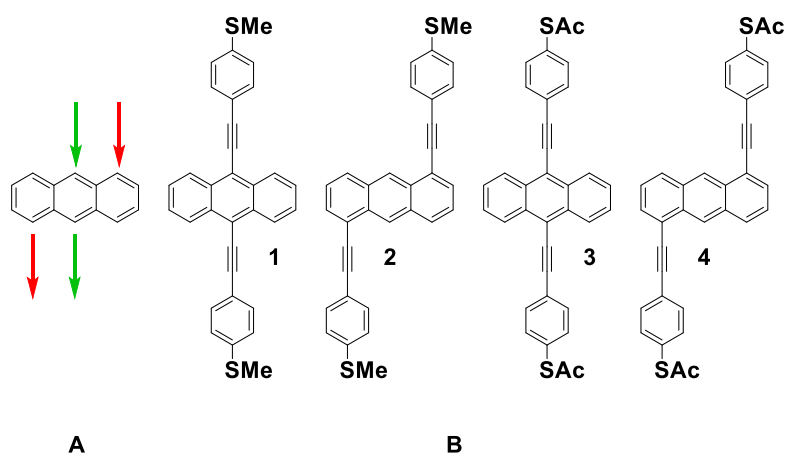

Fig. 1 | Structures of studied molecules. (A) A sketch of an anthracene core with connectivities $7,2^{\prime}$ and $1,5^{\prime}$. (B) Chemical realisations of molecular wires with anthracene cores. 1 and 3 correspond to the 7,2' connectivity, while 2 and 4 correspond to the $1,5^{\prime}$ connectivity.

Such a change in connectivity in a classical resistor network would lead to only a small change in electrical conductance. In contrast, theory predicts and experiment confirms $^{27-29}$ that the room temperature, single-molecule, low-bias electrical conductance $G_{1}$ for the green connectivity is approximately an order of magnitude greater than the conductance $G_{2}$ of the red connectivity. This is a clear signature of room-temperature phase-coherent transport and of the varying degrees of CQI for the two different 
connectivities. The chemical realization of the green connectivity is molecule $\mathbf{1}$ of Fig. 1 , in which the terminal groups attached to electrodes inject a current into the anthracene core via alkyne linkages. Similarly, molecule $\mathbf{2}$ is a realization of the red connectivity. 3 and 4 are alternative realisations of the red and green connectivities, in which the thioether terminal groups are replaced by thioacetate groups (which can be deprotected in-situ to grant terminal thiols for gold binding). These terminal anchor groups were chosen to demonstrate that further control over interfacial coupling and energy level alignment between molecules and electrodes could be achieved..$^{30-31}$ Our aim is to create self-assembled monolayers (SAMs) from these compounds, demonstrate that these singlemolecule signatures of CQI can be translated into SAMbased devices and assess the effect of CQI on their Seebeck coefficients. We indeed find that the electrical conductances of SAMs formed from $\mathbf{1}$ and $\mathbf{3}$ are significantly higher than those of SAMs formed from 2 and 4 . We also measure and calculate the Seebeck coefficients of these SAMs and show that the sign and magnitude of their thermopower is determined by a combination of their connectivities and the nature of their (thiolate or thioether) anchor groups. It should be noted that whilst thiol groups generally lead to stronger binding and superior film stability than thioethers, ${ }^{32}$ the latter are preferential where intermolecular interactions within the SAM may result in monolayer reorganization during assembly. $33-34$

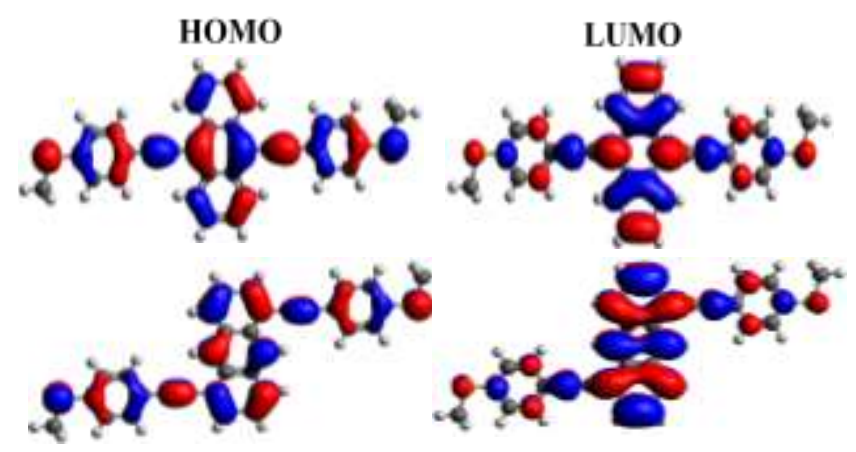

Fig. 2 | Frontier orbitals for 1 and 2: HOMO and LUMO orbitals for molecule 1 (top) and molecule 2 (bottom). (Orbitals for 3 and 4 are shown in the SI) Red (blue) corresponds to regions in space of positive (negative) orbital amplitude.

Our choice of connectivities in Fig. 1 was guided by 'magic ratio theory, ${ }^{, 27}$ which predicts that the ratio $\frac{G_{1}}{G_{2}}$ of the low-bias, single-molecule conductances of 1 and 2 (3 and 4) should be $\frac{G_{1}}{G_{2}}=16$ (ESI-Fig. S28). This simple theory illustrates how connectivity alone contributes to conductance ratios, without including chemical effects or Coulomb interactions. When the latter are included, recent studies 35 indicate that the qualitative trend in the ratio is preserved (i.e. that $\frac{G_{1}}{G_{2}} \gg 1$ ), but the precise value should be calculated using $a b$ initio methods. Our aim is to determine if this single-molecule signature of QI is preserved or modified in a SAM, where intermolecular interactions are also expected to play a role.

Fig. $\mathbf{2}$ shows the frontier orbitals of $\mathbf{1}$ and $\mathbf{2}$, and in agreement with magic ratio theory, confirms the presence of CQI, which occurs when the HOMO has different colours (representing different amplitudes) at the ends of the molecule (i.e. blue at one end and red at the other) and the LUMO has the same colour (i.e. red at both ends) ${ }^{29,36-38}$

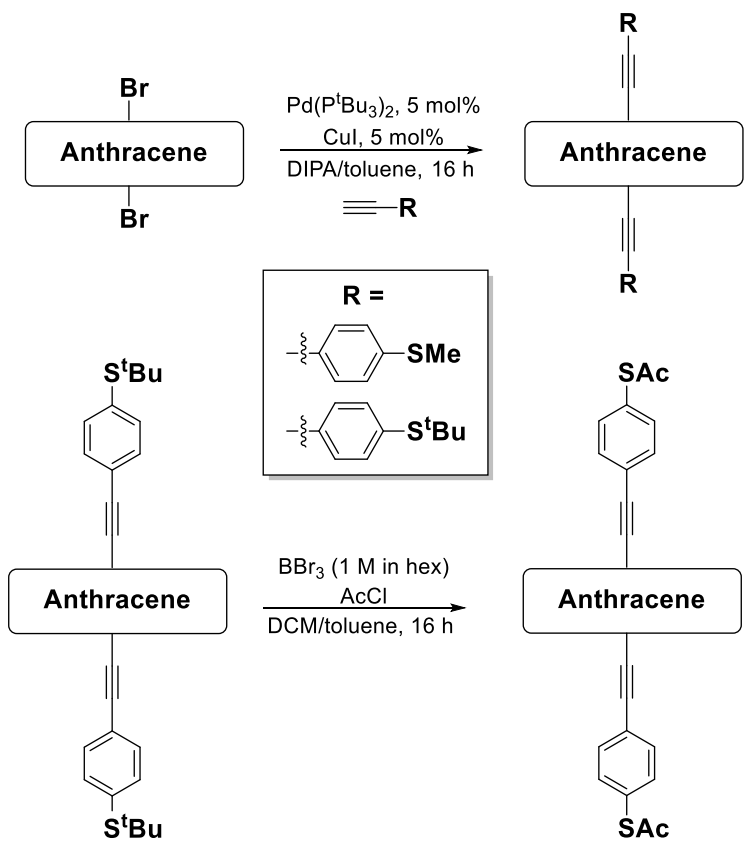

Scheme 1 | Synthesis of studied molecules. A representative synthetic pathway illustrating the construction of symmetric anthracenes through the use of Sonagashira (top) and trans-protection (bottom) reactions.

Molecules $\mathbf{1}$ and $\mathbf{2}$ bearing thioether termini could be synthesised from bromoanthracenes through the use of standard Sonagashira chemistry, however this same strategy could not be used to synthesise the thioacetate derivatives ( 3 and $\mathbf{4})$. This is due to a competing cyclo-oligermisation reaction that occurs when reacting a thioacetate-terminated phenylacetylide moiety in the presence of a palladium catalyst. 39 As a result of this, a trans-protection strategy was employed utilising a tert-butyl protected thiol. Initially, dibromoanthracenes were reacted with the alkyne of choice (either 4-ethynyl-tert-butylthioether or 4-ethynylthioanisole) to generate symmetrically disubstituted products $(1,2,3 \mathrm{~A}$ and $4 \mathrm{~A})$. All compounds could be purified via flash column chromatography and were obtained in good yields $(>60 \%)$. Thioacetate substituted anthracenes ( 3 and 4 ) were then obtained through transprotection reactions of $3 \mathrm{~A}$ and $4 \mathrm{~A}$ respectively. Molecule 4 could be purified through the use of flash chromatography alone, however recrystallization was required to isolate molecule 3, resulting in a slightly reduced yield (see SI 1.3).

Deposited molecular films were characterized by atomic force microscopy (AFM), nano-scratching ${ }^{40-42}$ and 
polished Au-coated quartz crystal microbalance (QCM), which suggested the formation of high-uniformity SAMs with thicknesses in the range of 1.1-1.4 $\mathrm{nm}$ (ESI Table $\mathrm{S}_{5}$ ); corresponding to a monolayer of molecules in a perpendicular configuration with a tilt angle of $30^{\circ}-50^{\circ}$. $43^{-44}$ All molecular films were grown on freshly prepared template stripped Au substrates ${ }^{45-46}$ with a surface roughness of $80-$ $150 \mathrm{pm}$ (see Methods section). Molecular conductance was characterized by conductive AFM (cAFM), where the number of molecules under the probe is estimated from the contact area between probe and sample surface (obtained via Hertz Model 47-49) and the single-molecule occupation area obtained from QCM and AFM.
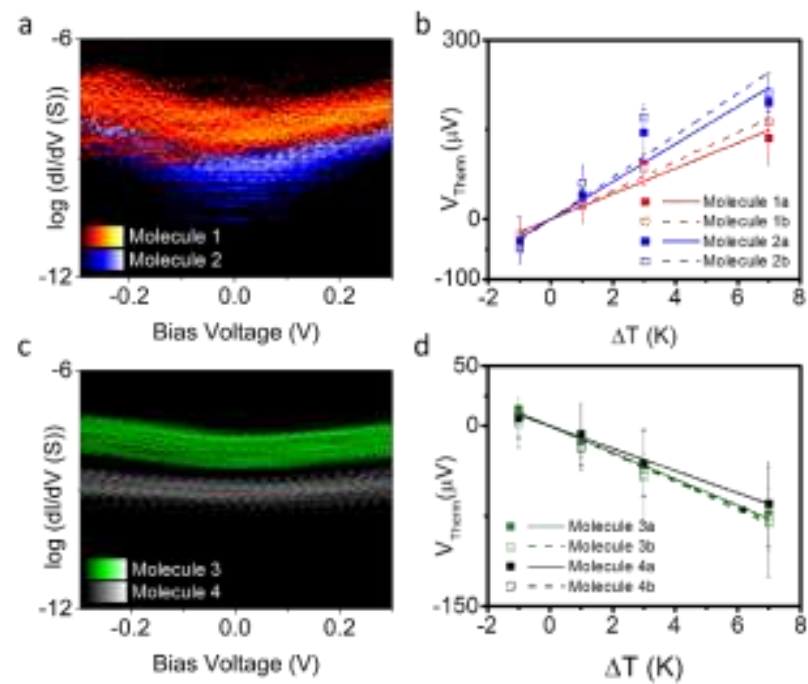

Fig. 3 | Electrical and thermoelectrical properties of SAMs. (a,c) Aggregate conductance vs voltage histogram of molecular conductance (molecule 1, 2 (a), and 3, 4 (c)), bias voltage between $-0.3 \mathrm{~V}$ to $-\mathbf{- 0 . 3} \mathrm{V}$. (b,d) Linear fit plot of Thermal Voltage vs. $\Delta T$ (Tsample - Tprobe) for molecules 1,2 (b) and 3,4 (d), SAMs Xa and SAMs Xb indicate two measured samples.

Aggregate conductance vs voltage histogram at low bias (-0.3 V to 0.3 V) for molecules 1-4 are shown in Figure za and c, while Figure $3 \mathrm{~b}$ and $d$ shows the linear fit of thermal voltage vs. $\Delta \mathrm{T}$ for different junction systems (see ESI Fig S 36 for detailed comparison of molecules 1 and 2). The slope of the fit, $\frac{V_{\text {Thermal }}}{\Delta T}$, related with the Seebeck coefficient of the junction via equation: $S_{\text {junction }}=S_{\text {probe-Au}}-$ $\frac{V_{\text {Thermal }}}{\Delta T}$ (the detailed number listed in Table 1). The Seebeck measurements of all SAMs were operated on two separate samples prepared with same recipe (labelled as SAMs Xa and SAMs Xb), and similar Seebeck coefficient values were obtained which confirmed the reliability of the measurement (Figure s37). The opposite slope of linear fit for $\frac{V_{T h e r m a l}}{\Delta T}$ between 1,2 and 3,4 demonstrating that the exchange of anchor groups leads to a change in sign for Seebeck coefficient.

From the statistics of $>\mathbf{2 0 0}$ different IV curves measured at different locations, the statistically-most-probable zero-bias differential conductance for molecule $\mathbf{1}$ is $\mathbf{1 0 . 2}$ times larger than that for molecule 2, and $\mathbf{1 4 . 2}$ times larger than that of molecule 3 .

To compute the electrical conductance of molecules 14, we use density functional theory combined with the quantum transport code Gollum ${ }^{50}$ to obtain the transmission coefficient $T(E)$ describing electrons of energy $E$ passing from the source to the drain electrodes, from which the room-temperature electrical conductance and Seebeck coefficient are determined.
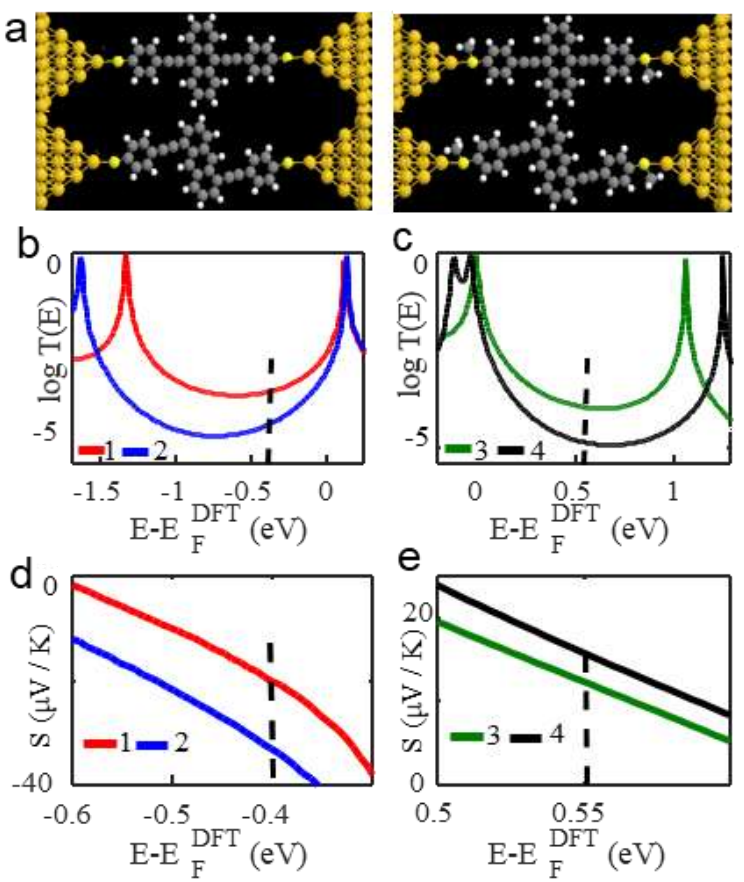

Fig. 4 | Charge transport in molecular junctions. (a) Schematic illustration of molecular junctions for $1,2,3$ and 4 . (b and c) Transmission functions $\mathbf{T}(\mathrm{E})$ for 1 (red solid-line), 2 (blue solid-line), 3 (green solid-line) and 4 (black solidline). (d and e ) Plots of the room-temperature Seebeck coefficients of 1-4 as a function of the Fermi energy $E_{F}$.

Fig. 4a shows that after structural relaxation, when placed between gold electrodes, the molecules adopt an angle corresponding to the measured tilt angle of the SAM (for different views see Fig. S27). It has recently been demonstrated, by comparing $\mathrm{T}(\mathrm{E})$ for a single molecule against SAMs consisting of 7 molecules, ${ }^{51}$ that the $T(E)$ for a SAM is approximately the same as for the single molecule. Fig. 4 b shows the computed transmission coefficients for all four junctions, while Figs. 4 d-e show the corresponding Seebeck coefficients as a function of the Fermi energy $E_{F}$. In agreement with previous studies, ${ }^{27}$ we find that the closest agreement between theory and experiment is obtained for a Fermi energy near the mid-gap, indicated by the vertical dashed lines in Figs. $4 \mathrm{~b}-\mathrm{c}$. The computed ratio of their transmission coefficients in goldmolecule-gold junctions (ESI-Fig. S28) for molecules 1 and 2 (similarly for 3 and 4 ) at $E=E_{F}^{\text {Gold }}$ is approximately 16. As described above, both molecules exhibit CQI near their gap centres and the conductance ratio arises from 
the different degrees of constructive QI associated with their different connectivities. ${ }^{36-37,52-57}$

When the terminal groups of molecules are changed from thioethers to thioacetates, the transmission coefficients for molecules 3 and $\mathbf{4}$ show the same trend as those associated with molecules $\mathbf{1}$ and $\mathbf{2}$ (see Table 1 ). The change in sign of the thermopower between terminal groups is due to the position of the frontier orbital energies relative to the Fermi energy of gold (Fig. 4); for the thioether-terminated molecules ( $\mathbf{1}$ and $\mathbf{2}$ ) the Fermi energy lies close to the LUMO, giving a positive slope and a negative Seebeck, whereas for the thiolate- (from thioacetate) terminated molecules ( 3 and 4 ) the HOMO is closer to the Fermi energy, giving a negative slope and a positive Seebeck.

Table 1 | Experimental measurements and standard uncertainty (std), and theoretical calculations $\left(E_{\mathrm{F}}-\mathrm{E}_{\mathrm{F}}{ }^{\mathrm{DFT}}=-\mathbf{0 . 4} \mathrm{eV}\right.$ for (1 and 2), $E_{F-} E_{F}{ }^{D F T}=+0.55 \mathrm{eV}$ for (3 and 4), average, yellow-lines in Fig S28 in the ESI)

\begin{tabular}{lllllll} 
M & Exp. & std & Theo. & Exp. S & std & Theo. S \\
& $(\mathbf{G} / \mathbf{G o})$ & & $(\mathbf{G} / \mathbf{G o})$ & $(\mu \mathrm{V} / \mathbf{K})$ & & $(\mu \mathrm{V} / \mathbf{K})$ \\
& & & & & & \\
\hline $\mathbf{1}$ & $7.01 \mathrm{E}-5$ & $9 \mathrm{E}-6$ & $1.66 \mathrm{E}-4$ & -23.4 & 4.6 & -20.0 \\
& & & & & & \\
\hline $\mathbf{2}$ & $6.88 \mathrm{E}-6$ & $1 \mathrm{E}-6$ & $1.05 \mathrm{E}-5$ & -31.8 & 6.1 & -33.0 \\
& & & & & & \\
\hline $\mathbf{3}$ & $1.28 \mathrm{E}-4$ & $5 \mathrm{E}-6$ & $1.59 \mathrm{E}-4$ & +12.1 & 3.0 & +12.5 \\
& & & & & & \\
\hline $\mathbf{4}$ & $9.0 \mathrm{E}-6$ & $3 \mathrm{E}-6$ & $1.00 \mathrm{E}-5$ & +10.4 & 1.1 & +16.3 \\
\hline
\end{tabular}

In summary, through the rational design, synthesis and implementation of a new family of molecules, we have demonstrated that unequivocal signatures of single-molecule room-temperature CQI, contained in the connectivity-dependent conductance ratio of 1 and 2 ( 3 and 4 ), can be translated into self-assembled molecular films. In contrast to previous work contrasting DQI with CQI effects in the Seebeck coefficient of a single molecule, ${ }^{14}$ here we have examined how different degrees of CQI can be used to control the thermopower of SAMs. Utilising CQI to control thermoelectricity is useful, since CQI allows the desirable possibility of high conductance, whereas DQI always leads to low conductance. With two different connectivities to the anthracene core, CQI effects lead to measured conductance ratios of $\left(G_{1} / G_{2}\right)_{\text {Exp. }}=10.2,\left(G_{3} / G_{4}\right)_{E x p \text {. }}=14.3$, for SAMs formed from 1 compared to 2 ( 3 and 4 ), which is comparable with the magic ratio of 16 and the single-molecule DFT values of $\left(G_{1} / G_{2}\right)_{\text {Theo. }}=15.8,\left(G_{3} / G_{4}\right)_{\text {Theo. }}=16.0$. Furthermore, we show that the thermoelectrical performance of anthracene-based molecular films can be boosted by a judicious choice of connectivity to electrodes, combined with an optimal choice of terminal groups. Although the effect of CQI on the electrical conductance of SAMs was reported only recently ${ }^{5}$, the above demonstration of CQI-controlled molecular films is the first report of CQIboosted thermoelectricity. It opens the way to new design strategies for functional ultra-thin-film thermoelectric materials and electronic building blocks for future integrated circuits.

\section{ASSOCIATED CONTENT}

Supporting Information is provided which contains all experimental details including information about the synthesis of the molecules, device fabrication and characterisation and the theoretical demonstration of molecular orbitals as well as the calculated transmission coefficient of gold/molecule/gold systems for all molecules. The Supporting Information is available free of charge on the ACS Publications website at DOI: ****

\section{AUTHOR INFORMATION}

Correspondence and requests for materials should be addressed to Professor Colin Lambert (c.lambert@lancaster.ac.uk), Professor Lesley Cohen (1.cohen@imperial.ac.uk), Professor Nicholas Long (n.long@imperial.ac.uk) or Dr. Benjamin Robinson (b.j.robinson@lancaster.ac.uk)

\section{ACKNOWLEDGMENTS}

Support from the UK EPSRC is acknowledged, through grant nos. EP/No17188/1, EP/Mo14452/1, EP/Po27156/1 and $\mathrm{EP} /$ No3337X/1. Support from the European Commission is provided by the FET Open project 767187 - QuIET. A.I is grateful for financial assistance from Tikrit University (Iraq), and the Iraqi Ministry of Higher Education (SL-20). NL is grateful for a Royal Society Wolfson Research Merit Award. LC and X.W acknowledge FSRF funding.

\section{REFERENCES}

1. Aradhya, S. V.; Venkataraman, L., Single-molecule junctions beyond electronic transport. Nat. Nanotech. 2013, 8 (6), 399-410.

2. Lambert, C., Basic concepts of quantum interference and electron transport in single-molecule electronics. Chem. Soc. Rev. 2015, 44 (4), 875-888.

3. Xiang, D.; Wang, X.; Jia, C.; Lee, T.; Guo, X., Molecularscale electronics: from concept to function. Chem. Rev. 2016, 116 (7), 4318-4440.

4. Jia, C.; Migliore, A.; Xin, N.; Huang, S.; Wang, J.; Yang, Q.; Wang, S.; Chen, H.; Wang, D.; Feng, B., Covalently bonded single-molecule junctions with stable and reversible photoswitched conductivity. Science 2016, 352 (6292), 14431445 .

5. Papadopoulos, T.; Grace, I.; Lambert, C., Control of electron transport through Fano resonances in molecular wires. Phys. Rev. B: Condens. Matter Mater. Phys. 20o6, 74 (19), 193306.

6. Markussen, T.; Schiotz, J.; Thygesen, K. S., Electrochemical control of quantum interference in anthraquinone-based molecular switches. J. Chem. Phys. 2010, 132 (22), 224104. 7. Vazquez, H.; Skouta, R.; Schneebeli, S.; Kamenetska, M.; Breslow, R.; Venkataraman, L.; Hybertsen, M., Probing the 
conductance superposition law in single-molecule circuits with parallel paths. Nat. Nanotech. 2012, 7 (10), 663-667. 8. Ballmann, S.; Hartle, R.; Coto, P. B.; Elbing, M.; Mayor, M.; Bryce, M. R.; Thoss, M.; Weber, H. B., Experimental evidence for quantum interference and vibrationally induced decoherence in single-molecule junctions. Phys. Rev. Lett. 2012, 109 (5), 056801.

9. Aradhya, S. V.; Meisner, J. S.; Krikorian, M.; Ahn, S.; Parameswaran, R.; Steigerwald, M. L.; Nuckolls, C.; Venkataraman, L., Dissecting contact mechanics from quantum interference in single-molecule junctions of stilbene derivatives. Nano Lett. 2012, 12 (3), 1643-1647. 10. Kaliginedi, V.; Moreno-Garcia, P.; Valkenier, H.; Hong, W.; Garcia-Suarez, V. M.; Buiter, P.; Otten, J. L.; Hummelen, J. C.; Lambert, C. J.; Wandlowski, T., Correlations between molecular structure and single-junction conductance: a case study with oligo(phenylene-ethynylene)-type wires. J. Am. Chem. Soc. 2012, 134 (11), 5262-5275.

11. Arroyo, C. R.; Tarkuc, S.; Frisenda, R.; Seldenthuis, J. S.; Woerde, C. H.; Eelkema, R.; Grozema, F. C.; van der Zant, H. S., Signatures of quantum interference effects on charge transport through a single benzene ring. Angew. Chem. 2013, 125, (11), 3234-3237.

12. Ke, S.-H.; Yang, W.; Baranger, H. U., Quantum-

interference-controlled molecular electronics. Nano Lett. 2008, 8 (10), 3257-3261.

13. Guédon, C. M.; Valkenier, H.; Markussen, T.; Thygesen, K. S.; Hummelen, J. C.; Van Der Molen, S. J., Observation of quantum interference in molecular charge transport. Nat. Nanotech. 2012, 7 (5), 305-309.

14. Miao, R.; Xu, H.; Skripnik, M.; Cui, L.; Wang, K.; Pedersen, K. G. L.; Leijnse, M.; Pauly, F.; Wärnmark, K.; Meyhofer, E.; Reddy, P.; Linke, H., Influence of Quantum Interference on the Thermoelectric Properties of Molecular Junctions. Nano Lett. 2018, 18 (9), 5666-5672.

15. Jia, C.; Famili, M.; Carlotti, M.; Liu, Y.; Wang, P.; Grace, I. M.; Feng, Z.; Wang, Y.; Zhao, Z.; Ding, M.; Xu, X.; Wang, C.; Lee, S.-J.; Huang, Y.; Chiechi, R. C.; Lambert, C. J.; Duan, X., Quantum interference mediated vertical molecular tunneling transistors. Science Advances 2018, 4 (10), eaat8237.

16. Fracasso, D.; Valkenier, H.; Hummelen, J. C.; Solomon, G. C.; Chiechi, R. C., Evidence for Quantum Interference in SAMs of Arylethynylene Thiolates in Tunneling Junctions with Eutectic Ga-In (EGaIn) Top-Contacts. J. Am. Chem. Soc. 2011, 133 (24), 9556-9563.

17. Carlotti, M.; Kovalchuk, A.; Wächter, T.; Qiu, X.;

Zharnikov, M.; Chiechi, R. C., Conformation-driven quantum interference effects mediated by through-space conjugation in self-assembled monolayers. Nat. Commun 2016, 7 (1), 13904.

18. Zhang, Y.; Ye, G.; Soni, S.; Qiu, X.; Krijger, Theodorus L.; Jonkman, H. T.; Carlotti, M.; Sauter, E.; Zharnikov, M.; Chiechi, R. C., Controlling destructive quantum interference in tunneling junctions comprising self-assembled monolayers via bond topology and functional groups. Chemical Science 2018, 9 (19), 4414-4423.

19. Carlotti, M.; Soni, S.; Kumar, S.; Ai, Y.; Sauter, E.; Zharnikov, M.; Chiechi, R. C., Two-Terminal Molecular Memory through Reversible Switching of Quantum Interference Features in Tunneling Junctions. Angew. Chem. Int. Ed. 2018, 57 (48), 15681-15685.

2o. Carlotti, M.; Soni, S.; Qiu, X.; Sauter, E.; Zharnikov, M.; Chiechi, R. C., Systematic experimental study of quantum interference effects in anthraquinoid molecular wires. Nanoscale Advances 2019, 1 (5), 2018-2028.

21. Rincón-García, L.; Evangeli, C.; Rubio-Bollinger, G.; Agraït, N., Thermopower measurements in molecular junctions. Chem. Soc. Rev. 2016, 45 (15), 4285-4306. 22. Rincón-García, L.; Ismael, A. K.; Evangeli, C.; Grace, I.; Rubio-Bollinger, G.; Porfyrakis, K.; Agraï, N.; Lambert, C. J., Molecular design and control of fullerene-based bithermoelectric materials. Nat. Mater. 2015, 15, 289. 23. Al-Khaykanee, M. K.; Ismael, A. K.; Grace, I.; Lambert, C. J., Oscillating Seebeck coefficients in $\pi$-stacked molecular junctions. RSC Advances 2018, 8 (44), 24711-24715.

24. Ismael, A. K.; Grace, I.; Lambert, C. J., Increasing the thermopower of crown-ether-bridged anthraquinones. Nanoscale 2015, 7 (41), 17338-17342.

25. Cui, L.; Miao, R.; Jiang, C.; Meyhofer, E.; Reddy, P., Perspective: Thermal and thermoelectric transport in molecular junctions. J. Chem. Phys. 2017, 146 (9), 092201. 26. Yzambart, G.; Rincón-García, L.; Al-Jobory, A. A.; Ismael, A. K.; Rubio-Bollinger, G.; Lambert, C. J.; Agraït, N.; Bryce, M. R., Thermoelectric Properties of 2,7-Dipyridylfluorene Derivatives in Single-Molecule Junctions. The Journal of Physical Chemistry C 2018, 122 (48), 27198-27204.

27. Geng, Y.; Sangtarash, S.; Huang, C.; Sadeghi, H.; Fu, Y.; Hong, W.; Wandlowski, T.; Decurtins, S.; Lambert, C. J.; Liu, S. X., Magic ratios for connectivity-driven electrical conductance of graphene-like molecules. J. Am. Chem. Soc. 2015, 137 (13), 4469-4476.

28. Sangtarash, S.; Huang, C.; Sadeghi, H.; Sorohhov, G.; Hauser, J.; Wandlowski, T.; Hong, W.; Decurtins, S.; Liu, S. X.; Lambert, C. J., Searching the Hearts of Graphene-like Molecules for Simplicity, Sensitivity, and Logic. J. Am. Chem. Soc. 2015, 137 (35), 11425-11431.

29. Lambert, C. J.; Liu, S. X., A Magic Ratio Rule for Beginners: A Chemist's Guide to Quantum Interference in Molecules. Chem. Eur. J. 2018, 24 (17), 4193-4201. 30. Jia, C.; Guo, X., Molecule-electrode interfaces in molecular electronic devices. Chem. Soc. Rev. 2013, 42 (13), 5642-566o.

31. Hong, W.; Manrique, D. Z.; Moreno-Garcia, P.; Gulcur, M.; Mishchenko, A.; Lambert, C. J.; Bryce, M. R.;

Wandlowski, T., Single molecular conductance of tolanes: experimental and theoretical study on the junction evolution dependent on the anchoring group. J. Am. Chem. Soc. 2012, 134 (4), 2292-2304.

32. Weidner, T.; Ballav, N.; Siemeling, U.; Troegel, D.; Walter, T.; Tacke, R.; Castner, D. G.; Zharnikov, M., Tripodal Binding Units for Self-Assembled Monolayers on Gold: A Comparison of Thiol and Thioether Headgroups. The Journal of Physical Chemistry C 2009, 113 (45), 19609-19617.

33. Piotrowski, P.; Pawłowska, J.; Pawłowski, J.; Czerwonka, A. M.; Bilewicz, R.; Kaim, A., Self-assembly of thioether functionalized fullerenes on gold and their activity in electropolymerization of styrene. RSC Advances 2015, 5 (105), 86771-86778.

34. del Carmen Gimenez-Lopez, M.; Räisänen, M. T.; Chamberlain, T. W.; Weber, U.; Lebedeva, M.; Rance, G. A.; Briggs, G. A. D.; Pettifor, D.; Burlakov, V.; Buck, M.; Khlobystov, A. N., Functionalized Fullerenes in SelfAssembled Monolayers. Langmuir 2011, 27 (17), 10977-10985. 35. Ulčakar, L.; Rejec, T.; Kokalj, J.; Sangtarash, S.; Sadeghi, H.; Ramšak, A.; Jefferson, J. H.; Lambert, C. J., On the 
resilience of magic number theory for conductance ratios of aromatic molecules. Sci Rep 2019, 9 (1), 3478.

36. Tsuji, Y.; Staykov, A.; Yoshizawa, K., Orbital views of molecular conductance perturbed by anchor units. J. Am. Chem. Soc. 2011, 133 (15), 5955-5965.

37. Yoshizawa, K.; Tada, T.; Staykov, A., Orbital views of the electron transport in molecular devices. J. Am. Chem. Soc. 2008, 130 (29), 9406-9413.

38. Li, X.; Staykov, A.; Yoshizawa, K., Orbital Views of the Electron Transport through Polycyclic Aromatic

Hydrocarbons with Different Molecular Sizes and Edge Type Structures. The Journal of Physical Chemistry C 2010, 114 (21), 9997-10003.

39. Inkpen, M. S.; White, A. J. P.; Albrecht, T.; Long, N. J., Avoiding problem reactions at the ferrocenyl-alkyne motif: a convenient synthesis of model, redox-active complexes for molecular electronics. Dalton Transactions 2014, 43 (41), 15287-15290.

40. Garcia, R.; Martinez, R. V.; Martinez, J., Nano-chemistry and scanning probe nanolithographies. Chem. Soc. Rev. 2oo6, 35 (1), 29-38.

41. Amro, N. A.; Xu, S.; Liu, G. Y., Patterning surfaces using tip-directed displacement and self-assembly. Langmuir 2ooo, 16 (7), 3006-3009.

42. Kaholek, M.; Lee, W. K.; LaMattina, B.; Caster, K. C.; Zauscher, S., Fabrication of stimulus-responsive nanopatterned polymer brushes by scanning-probe lithography. Nano Lett. 2004, 4 (2), 373-376.

43. Orata, D.; Buttry, D. A., Determination of Ion Populations and Solvent Content as Functions of Redox State and $\mathrm{Ph}$ in Polyaniline. J. Am. Chem. Soc. 1987, 109 (12), 3574-3581. 44. Sauerbrey, G., Verwendung Von Schwingquarzen Zur Wagung Dunner Schichten Und Zur Mikrowagung. Z. Physik 1959, 155 (2), 206-222.

45. Weiss, E. A.; Kaufman, G. K.; Kriebel, J. K.; Li, Z.; Schalek, R.; Whitesides, G. M., Si/SiO2-Templated formation of ultraflat metal surfaces on glass, polymer, and solder supports: Their use as substrates for self-assembled monolayers. Langmuir 2007, 23 (19), 9686-9694. 46. Banner, L. T.; Richter, A.; Pinkhassik, E., Pinhole-free large-grained atomically smooth $\mathrm{Au}(111)$ substrates prepared by flame-annealed template stripping. Surf. Interface Anal. 2009, 41 (1), 49-55. 47. Burnham, N. A.; Colton, R. J.; Pollock, H. M., WorkFunction Anisotropies as an Origin of Long-Range Surface Forces - Reply. Phys. Rev. Lett. 1993, 70 (2), 247-247. 48. Weihs, T. P.; Nawaz, Z.; Jarvis, S. P.; Pethica, J. B., Limits of Imaging Resolution for Atomic Force Microscopy of Molecules. Appl. Phys. Lett. 1991, 59 (27), 3536-3538.
49. Gomar-Nadal, E.; Ramachandran, G. K.; Chen, F.; Burgin, T.; Rovira, C.; Amabilino, D. B.; Lindsay, S. M., Self-

assembled monolayers of tetrathiafulvalene derivatives on $\mathrm{Au}(111)$ : Organization and electrical properties. J. Phys. Chem. $B$ 2004, 108 (22), 7213-7218.

50. Ferrer, J.; Lambert, C. J.; García-Suárez, V. M.; Manrique, D. Z.; Visontai, D.; Oroszlany, L.; Rodríguez-Ferradás, R.;

Grace, I.; Bailey, S.; Gillemot, K., GOLLUM: a next-generation simulation tool for electron, thermal and spin transport. New Journal of Physics 2014, 16 (9), 093029.

51. Herrer, L.; Ismael, A.; Martín, S.; Milan, D. C.; Serrano, J. L.; Nichols, R. J.; Lambert, C.; Cea, P., Single molecule vs. large area design of molecular electronic devices incorporating an efficient 2-aminepyridine double anchoring group. Nanoscale 2019, 11 (34), 15871-1588o.

52. Coulson, C.; Rushbrooke, G. In Note on the method of molecular orbitals, Mathematical Proceedings of the Cambridge Philosophical Society, Cambridge University Press: 1940; pp 193-200.

53. Zhao, X.; Geskin, V.; Stadler, R., Destructive quantum interference in electron transport: A reconciliation of the molecular orbital and the atomic orbital perspective. J. Chem. Phys. 2017, 146 (9), 092308.

54. Lambert, C. J.; Liu, S. X., A Magic Ratio Rule for

Beginners: A Chemist's Guide to Quantum Interference in Molecules. Chemistry-A European Journal 2018, 24 (17), 41934201.

55. Garner, M. H.; Li, H.; Chen, Y.; Su, T. A.; Shangguan, Z.; Paley, D. W.; Liu, T.; Ng, F.; Li, H.; Xiao, S.; Nuckolls, C.; Venkataraman, L.; Solomon, G. C., Comprehensive suppression of single-molecule conductance using destructive $\sigma$-interference. Nature 2018, 558 (7710), 415-419. 56. Naghibi, S.; Ismael, A. K.; Vezzoli, A.; Al-Khaykanee, M. K.; Zheng, X.; Grace, I. M.; Bethell, D.; Higgins, S. J.; Lambert, C. J.; Nichols, R. J., Synthetic Control of Quantum Interference by Regulating Charge on a Single Atom in Heteroaromatic Molecular Junctions. The Journal of Physical Chemistry Letters 2019, 10 (20), 6419-6424.

57. Ismael, A. K.; Grace, I.; Lambert, C. J., Connectivity dependence of Fano resonances in single molecules. PCCP 2017, 19 (9), 6416-6421.

58. Famili, M.; Jia, C.; Liu, X.; Wang, P.; Grace, I. M.; Guo, J.; Liu, Y.; Feng, Z.; Wang, Y.; Zhao, Z.; Decurtins, S.; Häner, R.; Huang, Y.; Liu, S.-X.; Lambert, C. J.; Duan, X., Self-Assembled Molecular-Electronic Films Controlled by Room Temperature Quantum Interference. Chem 2019, 5 (2), 474484 . 
Insert Table of Contents artwork here

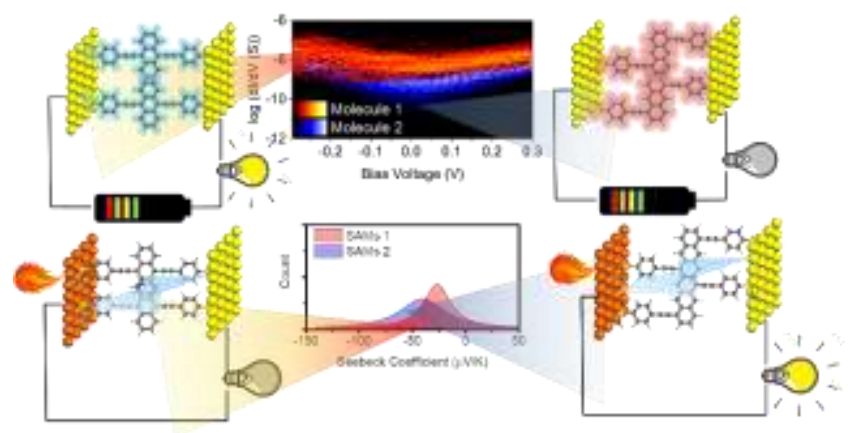

